\title{
SUFFICIENCY MODEL-ACTION CLARIFICATION FOR SIMULATION OPTIMIZATION APPLIED TO AN ELECTION SYSTEM
}

\author{
Anthony Afful-Dadzie \\ Theodore T. Allen \\ Alah Raqab \\ Jingsheng Li \\ 1971 Neil Avenue - 210 Baker Systems \\ The Ohio State University \\ Integrated Systems Engineering Department \\ Columbus, OH 43210 USA
}

\begin{abstract}
Many inputs for simulation optimization models are assumed to come from known distributions. When such distributions are obtained from small sample sizes, the parameters of these distributions may be associated with an "uncertainty set" or ranges. The presence of this uncertainty means that one or more solutions may be optimal depending on which parameters from the set are used. In this paper, we present a graphical methodology that combines bootstrap sampling and cross-evaluation techniques to visualize the data driven support for alternative solutions for problems in which distribution parameters are estimated using small sample sizes. We illustrate the methodology using a voting machine allocation problem.
\end{abstract}

\section{INTRODUCTION}

Simulation optimization techniques recommend design settings for systems that lack analytical expressions for input/output relations (Rosen, Harmonosky and Traband 2007). This is done through the evaluation of candidate solutions using a simulation model that takes candidate solutions as inputs, along with other parameter inputs based on fitted distributions ( $\mathrm{Fu} 2002)$.

In the presence of uncertainty in some of the inputs of the given simulation model (due to say, estimation of a distribution with smaller sample size), we can imagine that the model parameters derive from an "uncertainty set" which spans the values that are reasonably consistent with the available data. When this is the case, one or more solutions may be optimal depending on which parameters from the uncertainty set are used. It is both possible that solutions from different uncertainty set parameters may differ greatly in performance and the same solution may appear to foster vastly different performance if different parameters are applied (Delage and Mannor 2010, Dellino, Kleijnen and Meloni 2009). Under such circumstances, the objective of the modeler may shift to finding a solution that performs well across the many scenarios characterizing the uncertainty set.

Research on model uncertainty in simulation optimization is limited and focuses primarily on methods to obtain a single model-robust solution. Dellino, Kleijnen and Meloni (2009) consider solution robustness in simulation optimization using meta-model optimization-based approaches to obtain solutions that are relatively insensitive to perturbations in model parameters. Rosen, Harmonosky, and Traband (2007) develop simulation optimization models for systems with multiple performance measures taking into account end user's risk preference and uncertainty in the search process for the best solution. In this paper, we take a different perspective. Instead of seeking a single solution, we attempt to present the user with a visual display of relevant alternative solutions and the degree of support from the data for each solution. Our graphical methodology, called Sufficiency Model-Action Clarification Simulation (SMACSIM), is a modification of the approaches presented in (Allen and Rajagopalan 2011) and (Afful-Dadzie 
2012) and combines bootstrap sampling which is used for assessing uncertainty in distribution parameters (Chernick 2008), and cross-evaluation to generate and compare candidate solutions graphically.

The remainder of the paper is organized as follows. Section 2 presents the proposed methodology. In Section 3, we apply the method to an election systems allocation problem. Section 4 presents conclusions and directions for future research.

\section{PROPOSED GRAPHICAL METHODOLOGY}

Figure 1 describes the steps of the proposed sufficiency model-action clarification simulation (SMACSIM) methodology for simulation optimization. We use the vector $n$ to denote the sample sizes for all fitted distributions relevant to our model.

\section{Sufficiency Model-Action Clarification Simulation (SMAC-SIM) Method}

Step 1: (Naïve modeling) Perform input analysis as usual given the available data. Store all estimated model parameters in the vector $U_{o}$. We refer to $U_{o}$ as the naïve model.

Step 2: (Bootstrap sampling) Sample $m$ datasets each characterized by the original dataset sample size, $n$. For each dataset, refit all the needed distributions to obtain the model parameter sets $U=$ $\left(U_{1}, U_{2}, \ldots, U_{m}\right)$.

Step 3: (Multiple optimizations) For each $i=1,2, \ldots, m$, perform simulation optimization to generate the associated optimal solution $\pi_{i}$ and the performance value $z_{i}$ using inputs from model $U_{i}$. The resulting solutions are written $\Pi=\left(\pi_{1}, \ldots, \pi_{m}\right)$.

Step 4: (Probability estimation) Identify the $q$ distinct solutions among the $m$ solutions from Step 3 and tabulate the fraction of models associated with each of the $q$ unique solutions. These fractions are Monte Carlo probability estimates that the associated solutions are derived from the bootstrap sampling distribution.

Step 5: (Cross evaluation) Evaluate each of the $q$ distinct solutions from Step 4 using all the $m$ models from Step 2 and new random seeds to derive $m$ fresh performance estimates. These are the Monte Carlo estimates for mean performance.

Step 6: (Visualization) Compare the $q$ distinct solutions using box-and-whisker plots based on the $m$ Monte Carlo estimates for each distinct solution from steps 4 and 5.

Figure 1: Steps of the sufficiency model-action clarification simulation (SMAC-SIM) methodology.

The procedure in Figure 1 is similar to the fractional factorial data analysis method in Allen and Rajagopalan (2011). In Allen and Rajagopalan (2011), a Bayesian mixture model was applied to derive the $m$ datasets which are the sub-models being mixed. The bootstrap sampling procedure in Step 2 is arguably simpler and more generally relevant. This follows because sampling from the naïve model is often easily accomplished without restriction to available conjugate prior model formulations.

\section{CASE STUDY: VOTING-MACHINE ALLOCATION PROBLEM}

In this section, we describe the case study example relating to voting machine allocation via simulation optimization in preparation for the illustration of the SMAC-SIM methodology in the next section. We begin by reviewing the literature related to voting machine allocation. Next, we overview the three location problem, describe the input data and fitted distributions, the model formulation, and the simulation optimization. 
Afful-Dadzie, Allen, Raqab, and Li

\subsection{Background Related to Voting Machine Allocation}

Long waiting times experienced in recent general elections have drawn attention to the efficient allocation of election resources across voting locations (Yang, Fry, and Kelton 2009). Reducing waiting times which are non-increasing in number of servers or machines is important because it is a major factor to voter turnout (Spencer and Markovits 2010; Mebane 2005; Stein and Vonnahme 2012).

Edelstein and Edelstein (2010) list a number of issues that makes voting-machine allocation challenging and difficult to solve. Like any queuing problem, factors such as number of machines, arrival rate, voting times and expected voter turnout all play a major role in estimating waiting times of voters at a location. The arrival rate to polling stations is difficult to estimate because it is random and non-stationary with peaks in early morning, lunch time and after work hours (Edelstein 2006; Spencer and Markovits 2010). There is also uncertainty in voting times and turnout rates which are major factors in the votingmachine allocation problem.

The most popular voting allocation method used by many election boards in the U.S is apparently the "proportional allocation" method in which resources are allocated in proportion to the expected number of voters at each location (Edelstein 2006). This method seems appropriate when the number of ballot issues is uniform across locations. When differences exist in the number of ballot issues across locations, it introduces differences in voting times and the proportionality method results in unequal waiting times across the locations (Yang, Fry, and Kelton 2009).

A number of authors including Allen and Bernshteyn (2006a), Allen and Bernshteyn (2006b), Yang, Fry, and Kelton (2009), and Yang, Allen, Fry, and Kelton (2013) used simulation optimization models to analyze voting-machine allocation problems but did not consider uncertainty in distributions of inputs in their simulations.

\subsection{Model Overview}

We include parametric uncertainty in our simulation but focus on a relatively small allocation problem involving only three locations for the purpose of illustrating SMAC-SIM. Others, including Yang, Allen, Fry, and Kelton (2013) consider much larger problems involving hundreds of locations and thousands of resources (machines). Further, we assume the polls are open 13 hours plus the time it takes until all voters in line at 7:30 pm have voted.

Once a voter arrives to an assigned location, the voter joins a single first in first out (FIFO) queue until a voting machine becomes available. The model also assumes that all available voting machines to be allocated are identical. Our implementation of the simulation model is in Excel VBA and builds on the code developed for Yang, Allen, Fry, and Kelton (2013). We refer to our resources as "voting machines" because our voting time data derived from direct recording equipment (DRE) machines and ballot from the 2008 Ohio presidential election.

\subsection{Data and Input Distributions}

Next, we describe the relevant data in detail needed to simulate the arrival and service processes. The three locations are associated with short, medium, and long ballot lengths respectively. We assume the location with the short ballot has 1,500 registered voters, the location with the medium ballot, 1,200 registered voters and the location with the long ballot, 2,000 registered voters. The data on voting times comes from a mock voting exercise conducted in July 2008 in Franklin County, Ohio as part of a study to analyze the impact of the number of ballot issues on expected voter waiting time.

The service or voting time data in Table 1 was collected using a usability data capture tool from a project conducted on behalf of the Franklin County Board of Elections. The service time is the time required by the voter while they are monopolizing the machine or resource. The study involved 30 female and 30 male of voting age who used actual equipment and ballots to vote in a mock election. In terms of voting experience, $20 \%$ of the participant had never voted before, $60 \%$ had voted between 1 and 4 times in previous elections, while $20 \%$ had voted in at least 4 presidential elections. $35 \%$ of the participants 
were African Americans with the rest made up of other races. In addition, $40 \%$ of the participants had a college degree. Each location was assigned 20 voters. Table 1 shows the voting times for each voter for each of the 3 locations.

Table 1: Voting times of 20 voters at 3 locations from a mock voting exercise

\begin{tabular}{cccc}
\hline Voter & Long & Medium & Short \\
\hline 1 & 6.88 & 14.89 & 6.94 \\
2 & 7.70 & 7.30 & 4.52 \\
3 & 4.18 & 7.36 & 4.46 \\
4 & 6.24 & 5.55 & 5.00 \\
5 & 12.79 & 14.83 & 6.47 \\
6 & 12.45 & 7.06 & 6.54 \\
7 & 18.73 & 5.58 & 5.81 \\
8 & 8.42 & 8.55 & 7.93 \\
9 & 8.88 & 16.35 & 4.94 \\
10 & 6.34 & 9.71 & 5.46 \\
11 & 8.56 & 4.61 & 3.84 \\
12 & 5.01 & 9.62 & 5.21 \\
13 & 8.43 & 7.72 & 3.68 \\
14 & 6.77 & 6.90 & 4.25 \\
15 & 20.05 & 12.10 & 6.48 \\
16 & 15.88 & 12.69 & 13.68 \\
17 & 10.90 & 9.35 & 7.95 \\
18 & 18.97 & 7.92 & 4.09 \\
19 & 12.91 & 12.01 & 7.33 \\
20 & 12.77 & 9.76 & 6.47 \\
\hline & & &
\end{tabular}

Following Yang, Allen, Fry, and Kelton (2013), we apply the lognormal distribution for modeling the service times. The lognormal distribution is expressed in terms of the parameters $\mu_{i}$ and $\sigma_{i}$ for locations $i$ $=1,2$, and 3 . Here, we estimate the parameters from our data using the maximum likelihood estimators of the lognormal distribution :

$$
\begin{array}{cc}
\hat{\mu}_{i}=\frac{\sum_{k} \operatorname{In} x_{k}}{n} & i=1,2,3 . \\
\widehat{\sigma}_{l}^{2}=\frac{\sum_{k}\left(\operatorname{In} x_{k}-\widehat{\mu}_{i}\right)^{2}}{n} & i=1,2,3 .
\end{array}
$$

The "naïve" estimates based on the data in Table 1 and the formulas in equations (1) and (2) are: $\hat{\mu}_{1}=$ 2.27, $\hat{\sigma}_{1}=1.51, \hat{\mu}_{2}=2.19, \hat{\sigma}_{2}=1.47, \hat{\mu}_{3}=1.75$, and $\hat{\sigma}_{3}=1.32$.

\subsection{The Min-Max Optimization Model}

The underlying optimization model in our simulation comes from the "minimax" optimization formulation from Yang, Allen, Fry and Kelton (2013) and Allen and Bernshteyn (2006a). Specifically, the objective is to choose a machine allocation that minimizes the maximum expected voter waiting times across all locations, written formally as:

$$
\min _{x_{i}} Z(X)
$$


subject to

$$
\begin{gathered}
\sum_{i=1}^{N} x_{i}=M \\
x_{i} \geq b_{i}, x_{i} \in\{1,2,3 \ldots\} \\
Z(X)=\max _{i \in N} W_{i}(X)
\end{gathered}
$$

where $N$ is the number of locations or polling stations, $M$ is the total number of available voting machines to be allocated, and $W_{i}(X), i \in N$ is the average waiting time in location $i . b_{i}$ is the minimum number of voting machines required at location $i$, and $x_{i}$ is a positive integer. We assume that $\sum_{i=1}^{N} b_{i} \leq M$ so that feasible solutions exist. Also, $X=\left(x_{1}, x_{2}, \ldots, x_{N}\right)^{\prime}, x_{i}(i \in N)$ is the number of voting machines allocated to location $i$, such that $w_{i}\left(x_{i}\right), i \in N$ is the waiting time at location $i$ with $x_{i}$ voting machines.

\subsection{Algorithm Description of the Min-Max Formulation}

To solve the simulation optimization problem, we apply the greedy constant sample size heuristic from Yang, Allen, Fry and Kelton (2013). To review, the heuristic solution method is:

Step 1. Assign initial values to $x_{i}$ for each location $i$ (by default set $x_{i}=1$ for all $i$ ). Counter $=\sum_{i=1}^{N} x_{i} \leq M$

Step 2. If Counter $=M$ stop. Otherwise go to Step 3 .

Step 3. Run the simulation with $r$ replications to generate estimates for the expected waiting times at each location. Let $x_{i}=x_{i}+1$ for the location $i$ with the largest estimated expected waiting time in queue. Set Counter $=$ Counter +1 , and go to Step 2 .

Yang, Allen, Fry and Kelton (2013) provide a rigorous guarantee for the solution quality associated with this heuristic computed at the time of termination.

\section{APPLICATION AND RESULTS}

In this section, we apply the SMAC-SIM method to the three location voting machine problem using three assumptions or cases relating to service (voting) time data availability. All three cases share the same inputs for the simulation model except data on voting times which differ in quantity. The first is based on the 20 data per location in Table 1 . The second and third are based on hypothetical cases in which 500 and 1,000 service times per location are available, respectively. We note here that, using only the naïve model for the simulation optimization leads to an optimal allocation of 37-23-15 representing 37 machines at the location with long ballot, 23 machines at the location with medium ballot, and 15 machines at the location with shorter ballot. The average of the expected waiting time across the 3 locations is 61.22 minutes, with a maximum of 67.14 minutes at the location with long ballot length.

\subsection{SMAC-SIM Application with 20 Data Points Per Location}

Data on voting times for the first case (Case \#1) is shown in Table 1 which is made up of 20 samples of voting times for each of the three locations. In Step 1, we fit a naïve distribution to the $n=20$ samples of voting times from Table 1 and call this, together with the other input distributions and parameters, model $U_{o}$. In Step 2, we generate $m=20$ models where for each model, the distribution parameter for the voting time is estimated from the 20 bootstrap samples of the naïve distribution from Step 1. In Step 3, we perform simulation optimization for each of the $m=20$ models to generate 20 optimal solutions. In Step 4, we identify that there are $q=7$ unique allocations and tabulate the probability estimates associated with the unique allocations. Step 5 evaluates each of the $q=7$ unique allocations using all the $m=20$ models and tabulates the 20 associated expected waiting time values for each unique allocation. In Step 6, multiple box-and-whisker plots are generated from the 20 expected waiting times for each of the $q=7$ unique allocations. The box-and-whisker plots are shown in Figure 2, ordered by the estimated probabilities from smallest to highest. 
In Figure 2, we see that allocations 37-23-15 and 34-23-18 were each suggested by 1 model (5\%) as the optimal allocation. The other allocations listed are supported by more than a single model resulting in higher estimate probabilities. For example, 4 models all generated the same allocation $36-21-18$ as putatively optimal.

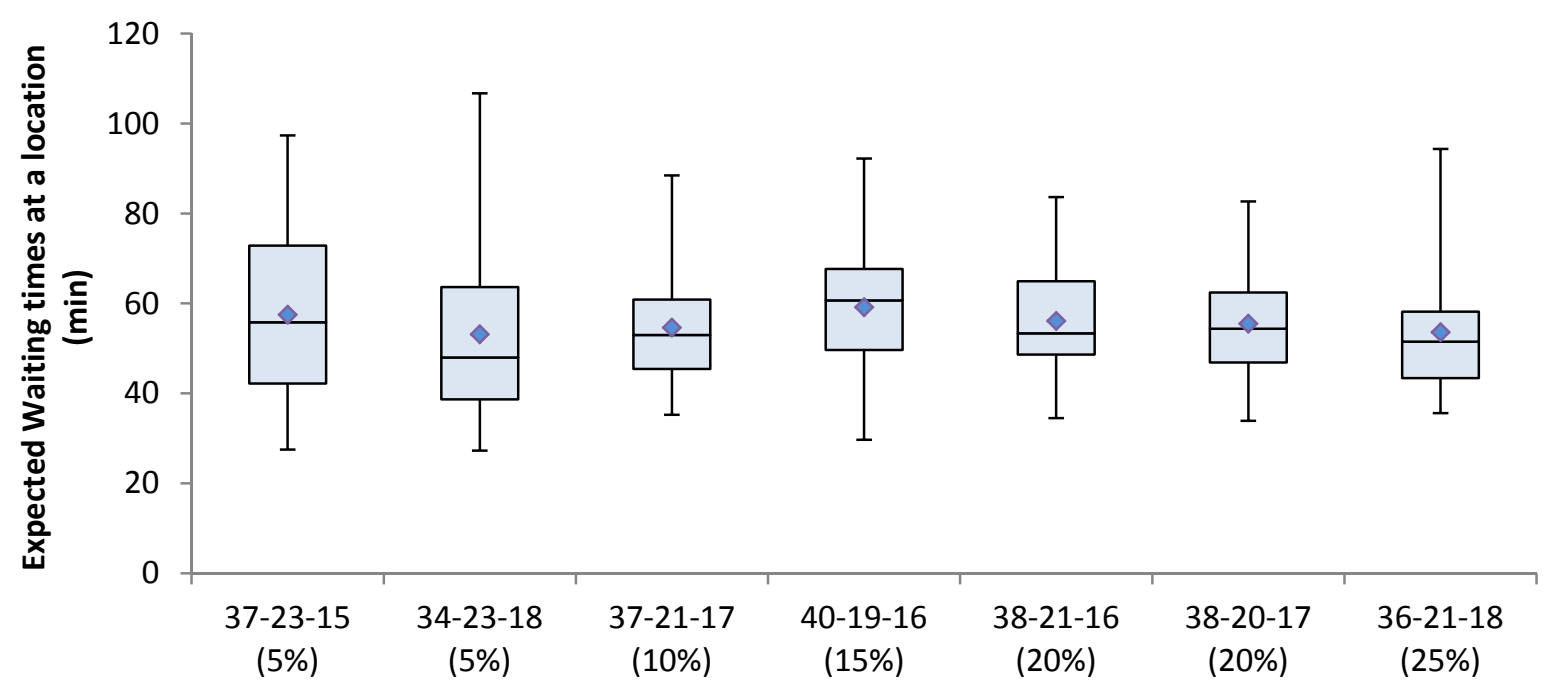

Machine Allocation and Estimated Probabilities

Figure 2: SMAC-SIM plots of expected waiting times for voting-machine allocation policies. The numbers separated by a hyphen represent the number of machines allocated to the 3 locations long, medium and short in the order given. For instance, allocation 37-23-15, will allocate 37 machines to location the location with long ballot, 23 to the location with medium ballot and 15 to the location with the shorter ballot. The accompanying percentage in bracket represents the number of models for which the allocation was optimal.

The box-and-whisker plots characterizes the expected mean performance for each allocation under all 20 models. For example, regardless of which model occurs in reality, the maximum expected waiting time under allocation 37-23-15 would not exceed 100 minutes, and the minimum expected waiting time would be as low as 30 minutes. One could choose to place more confidence in say allocation 38-20-17 than allocation 37-23-15 for the fact that allocation 38-20-17 has a shorter maximum expected waiting time and a tighter bound (of about 50 minutes) to allocation 37-23-15.

The width of the box-plot represent the estimated range of the effect of parameter uncertainty on expected waiting time that could be removed by additional data collection. Roughly about 25 minutes in uncertainty could be removed by additional data collection.

A large number of solutions on the $\mathrm{x}$-axis is an indication of high parametric uncertainty. When that happens it may be advisable to collect additional data to reduce the uncertainty (see section 4.2). Yet, if one or more of the optimal solutions is predicted to achieve desirable performance regardless of which model is accurate, one might logically recommend that allocation without further additional data collection.

Comparing the SMAC-SIM results in Figure 2 to the one from the naïve model, we see that, the performance of allocation 37-23-15 (which is also the allocation suggested by the naïve model) clearly shows a different picture than that from the naïve model. Here, we see that if the worst possible scenario was to occur, allocation 37-23-15 would lead to an expected waiting time of nearly 100 minutes at one of the 3 locations. This is in sharp contrast to the 67.14 minutes predicted by the naïve model.

In the next subsection, we examine how the number of optimal allocation (solution) choices as well as associated performance interval reduces as more data become available. 


\subsection{SMAC-SIM Application with 500 Data Points Per Location}

Next, we derive the SMAC-SIM plot for a hypothetical case in which the naïve model is the same but we imagine that the sample size which derived it was $n_{1}=n_{2}=n_{3}=500$. In Step 4 , we identify only $q=4$ unique allocations. The derived SMAC-SIM plot is shown in Figure 3. Note that with more data, allocation 37-21-17 is now suggested by $30 \%$ of the models compared to $10 \%$ from Case \#1 to be optimal. Allocation 37-23-15 (which is also suggested by the naïve model) is now not among the likely optimal models. Also, only 3 of the allocations from Case \#1 is now considered among the likely optimal allocations. Allocation 39-20-16 is not even suggested in Case \#1. We also see that the widths of the plots are now tighter signifying a reduction in uncertainty. From Figure 3, it look as if though the 4 allocations would perform approximately the same.

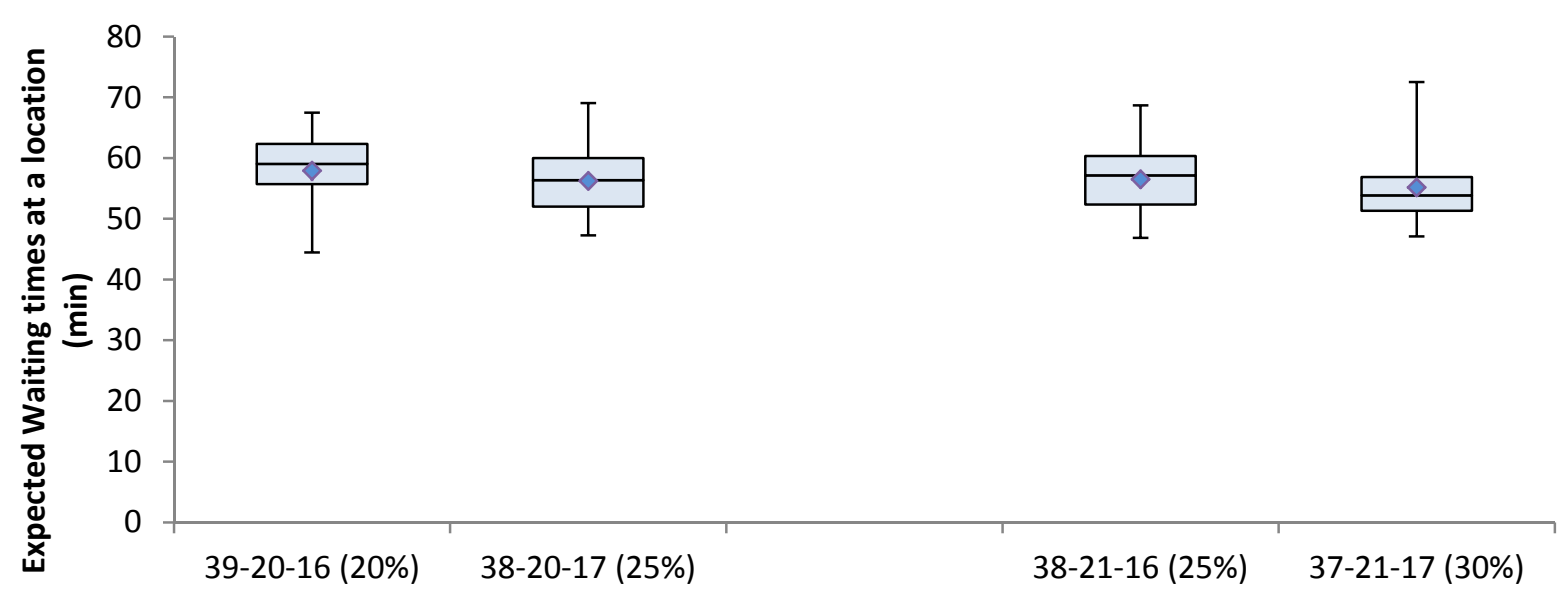

Machine Allocation and Estimated Probabilities

Figure 3: SMAC-SIM plots of expected waiting times for voting-machine allocation policies. The numbers separated by a hyphen represent the number of machines allocated to the 3 locations long, medium and short in the order given. For instance, allocation 39-20-16, will allocate 39 machines to the location with long ballot, 20 machines to the location with medium ballot and 16 machines to the location with shorter ballot. The accompanying percentage in bracket represents the number of models for which the allocation was optimal.

\subsection{SMAC-SIM Application with 1,000 Data Points Per Location}

Next, we derive the SMAC-SIM plot for a hypothetical case in which the naïve model is the same but we imagine that the sample size which derived it was $n_{1}=n_{2}=n_{3}=1,000$. Here, Step 4 identifies only $q=3$ policies. As shown in Figure 4, the optimality of allocation 37-21-17 is still not assured (only 55\% likely to be the optimal allocation) but the possible relative losses for accepting this allocation are likely minimal because of the narrow ranges in the simulated waiting times values from Step 5. Also, the loss in performance from employing allocation 38-21-16 over allocation 37-21-17 are predicted to be minimal, even while allocation 38-21-16 has only an 20\% likelihood of being optimal.

\section{CONCLUSION AND FUTURE WORK}

In this paper, we have presented a new methodology called Sufficiency Model-Action Clarification Simulation (SMAC-SIM) which provides insight about the impact of parametric uncertainty on the optimal solutions from simulation optimization models. Using box-and-whisker plots, the performance of multiple relevant optimal solutions and their estimated optimality probabilities are displayed. We illustrated the proposed method using a three location voting allocation problem. The results dramatize the limitation of 
using only 20 service time data points per location. The resulting uncertainties in the performance of the recommended allocations vary widely enough to, potentially, justify additional data collection. This follows because the ranges on the SMAC-SIM in Figure 2 might be considered unacceptably wide. For example, if the 36-21-18 allocation were applied, some of the relevant model supported by the data indicate expected waiting times of 100 minutes could be possible.

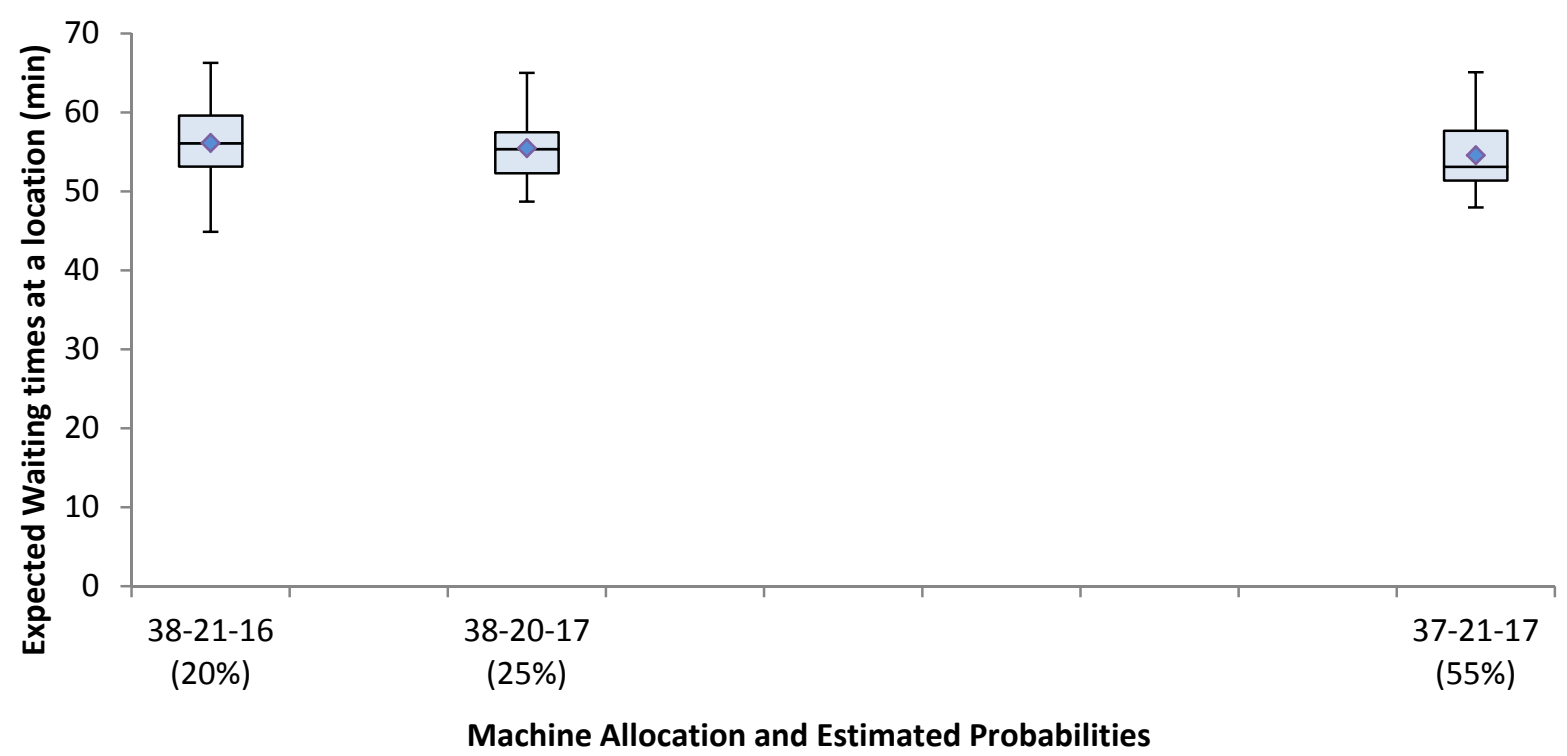

Figure 4: SMAC-SIM plots of expected waiting times for voting-machine allocation policies. The numbers separated by a hyphen represent the number of machines allocated to the 3 locations long, medium and short in the order given. For instance, allocation 38-21-16, will allocate 38 machines to the location with long ballot, 21 machines to the location with medium ballot and 16 machines to the location with shorter ballot. The accompanying percentage in bracket represents the number of models for which the allocation was optimal.

One benefit of the SMAC-SIM approach and the associated plots is that it could help the decision maker choose solutions that are in some sense, robust to any possible scenario. For example, from Figure 2 we conclude that the allocation 38-20-17 offers relatively good model robustness in that none of the 20 models derived from bootstrap sampling predict longer than 90 minutes of expected waiting times.

The SMAC-SIM approach and the derived plots also provide insight into the need for additional data collection and the amount of performance gains that could be derived from additional data. More solutions on the $\mathrm{x}$-axis is an indication of higher parameter uncertainty. As more data becomes available, the number of suggested solutions reduces and the difficulty in choosing among competing solutions lessons. When more data is also available, the width of the box plots becomes tighter and expected performance results becomes more definitive. The SMAC-SIM approach is fully automated. In particular, the process for determining the alternative optimal solutions is fully incorporated in the simulation optimization process.

A number of topics remain for future research. First, one could consider uncertainty in assumed parameters as well as distribution choices in addition to distribution parameters. Secondly, formulations that explicitly include data collection as an option can be developed. These can be attempted using for example, a Bayesian prior posterior formulation to capture the likely value of additional data collection. Thirdly, a shortcoming of the bootstrap sampling approach is that it does not provide general finite-sample guarantees. Therefore, alternative sampling approaches may be needed when sufficiently large samples cannot be obtained. One possible alternative to bootstrap sampling could be based on randomly dividing the input data into three or more groups, fitting models to each group and basing simulations off the de- 
rived parameters. The derived simulation outputs would be independent with respect to the randomness in the input data. Under approximate normality assumptions, policy options could then be evaluated with rigorous confidence intervals.

\section{ACKNOWLEDGEMENTS}

We thank Muer Yang for his code and support. We also thank the People's Republic of China for supporting Jingsheng Li as a visiting scholar.

\section{REFERENCES}

Afful-Dadzie, A. 2012. "Robust Optimal Maintenance Policies and Charts for Cyber Vulnerability Management." PhD. Dissertation.

Allen, T.T and M. Bernshteyn. 2006. "Mitigating voter waiting times." Chance 19(4): 25-36

Allen, T.T. and R. Rajagopalan. 2011. "A Bayesian Plotting Method for Fractional Factorial Data Analysis.” Journal of Quality Technology 43 (3): 224-236.

Chernick, M.R. 2008. "Bootstrap methods: A guide for practitioners and researchers." $2^{\text {nd }}$ ed. John Wiley \& Sons.

Delage, E., and S. Mannor. 2010. "Percentile optimization for Markov decision processes with parameter uncertainty." Operations Research, 58(1), 203-213.

Dellino, G., J. P. Kleijnen, and C. Meloni. 2009. "Robust simulation-optimization using metamodels." In Proceedings of the 2009 Winter Simulation Conference, ed. M.D. Rossetti, R.R. Hill, B. Johansson, A. Dunkin, and R.G. Ingalls, 540-550.

Edelstein, W. A., and A. D. Edelstein. 2010. "Queuing and elections: long lines, DREs and paper ballots." Proceedings of EVT/WOTE 2010.

$\mathrm{Fu}$, M. C. 2002. "Optimization for simulation: theory vs. practice.” INFORMS Journal on Computing, 14(3), 192-215.

Rosen, S. L., C. M., Harmonosky, and M. T. Traband. 2007. "A simulation optimization method that considers uncertainty and multiple performance measures." European Journal of Operational Research, 181(1), 315-330.

Spencer, D. M., and Z. S. Markovits. (2010). "Long lines at polling stations? Observations from an election day field study." Election Law Journal, 9(1), 3-17.

Stein, R. M., and G. Vonnahme. (2012). "Effect of Election Day Vote Centers on Voter Participation." Election Law Journal, 11(3), 291-301.

Yang, M., T. T. Allen, M. J. Fry, and W.D. Kelton. 2013. "The Call for Equity: Simulation-Optimization Models to Minimize the Range of Waiting Times." IIE Transactions 45 (7): 781-795.

Yang, M., M. J. Fry, and W.D. Kelton. 2009. "Are all voting queues created equal?" In Proceedings of the 2009 Winter Simulation Conference, ed. M.D. Rossetti, R.R. Hill, B. Johansson, A. Dunkin, and R.G. Ingalls, 3140-3149.

\section{AUTHOR BIOGRAPHIES}

Anthony Afful-Dadzie, Ph.D. is a postdoctoral researcher at the Integrated System Engineering department at The Ohio State University. He received his Ph.D. in industrial and systems engineering from The Ohio State University, his M.S. in economics from The Ohio State University, and his MPhil. in industrial systems, manufacturing, and management from University of Cambridge (U.K.). His interests include data mining and data analytics, quality control, engineering education, reliability engineering, and related optimization. His email address is afful-dadzie.1@osu.edu. 
Theodore T. Allen, Ph.D is an associate professor in the Department of Integrated Systems Engineering, College of Engineering at The Ohio State University. His interests focus on the overlap of operations research and quality improvement with emphasis on applied statistics and design of experiments (DOE). Allen has worked with numerous companies applying related techniques including as a full-time intern at The Ford Motor Company. He is a fellow of the American Society for Quality and the author of more than 40 peer-reviewed papers including two textbooks. His email address is allen.515@osu.edu.

Alah Raqab is graduate student (Master of Science) at Ohio State University in Industrial and Systems Engineering (ISE). He is also working as graduate teaching associate at ISE deportment. Raqab Graduated as an Industrial Engineer from the University of Jordan / Amman, he joined TAG-Consultants in 2011 and started at Amman office where he was working in Management \& industrial consulting. Raqab is an American Society for Quality Certified Six Sigma Green Belt, certified since December, 2011. He is also Quality Management System (ISO 9001) certified Lead Auditor, successfully completed the training courses conducted by SGS-Jordan in 2011. His email address is raqab.3@osu.edu.

Jingsheng Li is a visiting scholar in the Department of Integrated Systems Engineering at the Ohio State University. He is a PhD candidate in the Mechanical Engineering at Beijing Institute of Technology, China. His email is ljsbit@yahoo.cn. 